\title{
A Fuzzy Logic Approach to Changing Media Frames of Arafat and Sharon Following the Cataclysmic Events of Sept. 11, 2001
}

\author{
George E. Tsekouras ${ }^{1}$, Philemon Bantimaroudis*,1, and Susan Dente Ross ${ }^{2}$ \\ ${ }^{1}$ Department of Cultural Informatics, University of the Aegean; ${ }^{2}$ Department of English, Washington State University
}

\begin{abstract}
U.S. perceptions of the world shifted on Sept. 11, 2001. The changed U.S. understanding of the world clearly is tied to events. However, given the power of mediated messages of traumatic events to reshape media consumer's interpretation of reality, Americans post-Sept. 11 understanding also may be tied to changes in media presentations of news events and actors. To assess the extent to which U.S. media portrayals of the world changed after Sept. 11, the authors scrutinized one global elite newspaper's portrayals of two Middle East leaders engaged in chronic violence and acts of terror. Using categorical variables to represent different media frames and employing fuzzy logic cluster analysis, the authors determined that The New York Times' portrayal of Ariel Sharon and Yasser Arafat changed following the Sept. 11 catastrophe.
\end{abstract}

\section{INTRODUCTION}

The Sept. 11, 2001 terrorist offensive against the World Trade Center and the Pentagon belongs to a distinct category of events often described as catastrophic or cataclysmic because of their impact on people's perceptions, attitudes, and behaviors. Scholars argue that from such catastrophic events "new paradigms arise" (Savitch, 2003, p. 108). The current study posits that after the catastrophe of Sept. 11, 2001, a media reframing process was initiated related to media coverage connected to U.S. interests and policies around the globe.

Two Middle East leaders -- Yasser Arafat and Ariel Sharon - were chosen because of their significance for the U.S. foreign policy. Their long-standing and pivotal significance for U.S. foreign policy renders both of them useful for the current case study examining the possibility of a reframing process. Relevant theories also came under scrutiny.

Better understanding of media frame shifting tendencies carries significant implications for the public, politicians, and academics. Although media frames are relatively stable and convey a dominant ideology, they sometimes change in ways that may profoundly alter deeply held beliefs and attitudes and also may lead to new policies and initiatives (Ross \& Bantimaroudis, 2006).

\section{LITERATURE REVIEW}

Frames are interpretive schemata that are embedded in the narrative organization and the semantic content of messages (Snow \& Bedford, 1992). Framing is widely understood to result from the necessary selection of information by message makers. Selections are not random. Rather they reflect systemic structural, political, and cultural

*Address correspondence to this author at the Department of Cultural Informatics, University of the Aegean, P.O. Box 108, Mytilene, T.K. 81100, Greece; Tel: 0030-22510-31546; Office: 0030-22510-31620;

Fax: 0030-22510-36609; E-mail: pbantima@aegean.gr biases of news making in ways that inform the semantic content of messages. Media frames pervade content and endure because they are produced by deeply embedded, shared media norms, professional practices, and ideologies (Shoemaker, 1984). News frames convey a specific worldview, and the recurrence and reproduction of relatively stable frames across media and through time affects both what the audience thinks about, and how the audience thinks about those topics. For example, media frames reinforce the status quo from which the media and the broader societal elites benefit (Schlesinger, Elliot \& Murdock, 1984; Shoemaker, 1984; Steuter, 1990; Wolfsfeld, 1997).

Despite their relative stability, media frames do shift (Entman, 2004).

Media act either as agents of social control or social change, depending upon the issues and events driving coverage, and the news organization's resources, political culture, and dependency on government sources (Wolfsfeld, 1997; Shin, et al., 1999). Political opportunity and crisis theories suggest that social redefinition of groups or issues generally requires a major catalyzing event to galvanize public attention and provide an opportunity for change (Rosenthal, 2003). Similarly, some research suggests that shifts in media frames require a dramatic event or a critical discourse moment (Gamson, 1992; Kepplinger \& Habermeier, 1995; Berkowitz, 1992). Dramatic events that drive altered news coverage patterns and content decisions are correlated with alterations in the nature of the media content.

The Sept. 11, 2001 terrorist offensive against the World Trade Center and Pentagon has been described as catastrophic or cataclysmic because of its impact on people's perceptions, attitudes, and behaviors. Given that such catastrophic events prompt "new paradigms" (Savitch, 2003), one might expect the crisis of Sept. 11, 2001, to trigger media reframing. However, scholars have not fully explored when and why media frames shift or the stability of transformed frames. Some work suggests that significant frame shifts may be both superficial and transient (Mandelzis, 2003). Much work remains to explicate media reframing of issues and 
actors. Of central importance to frame shifting research is whether reframing is permanent or whether frames rebound to their initial state or settle on some new representation.

\section{METHODOLOGY}

The central hypothesis of this study is that following the Sept. 11 attacks, U.S. media likely would reframe news content that previously emphasized congruent themes and issues closely associated with the events of Sept. 11 (Norris, Kern \& Just, 2003). Because the Sept. 11 attacks were framed as an episode of global terrorism involving the United States (Entman, 2004), the authors anticipated media reframing would occur in news coverage of worldwide terrorism and terrorist groups that implicated U.S. interests (Ross, \& Bantimaroudis, 2006).

To verify the above hypothesis, the current study posits that the catastrophe of Sept. 11, 2001, initiated a media reframing process related to news coverage connected to U.S. interests and policies around the globe. We chose to test this theory by examining a topic long framed by U.S. media in terms of terrorism: the Israeli/Palestinian conflict and particularly the respective leaders of that time-period namely, Ariel Sharon and Yasser Arafat. The ongoing conflict between Israel and Palestine is a relevant case study because of the strong link between the Middle East and vital U.S. interests of strategic significance. Examination of frame shifting logically starts with elite international media. Therefore, the authors conducted a content analysis of The New York Times, which is a global elite medium with a worldwide influence (Hess, 1996; Reese \& Danielian, 1991). Researchers routinely describe The New York Times as an elite medium, influencing the content of other national and regional media (Shah \& Thornton, 2004).

\section{Data Collection and Description}

Based on a broad review of the literature, explicit references to the two leaders were coded for a wide range of previously identified affective and cognitive attributes most salient and congruent to the cataclysmic event of Sept. 11. Coding assessed the tone of the media coverage and measured variables such as each leader's authority and alignment with U.S. policies.

To conduct the content analysis, two experienced framing coders searched the file of The New York Times in the NEXIS database for all references to Sharon and/or Arafat between 3/1/2001 and 2/28/2002 (i.e., the twelve months surrounding Sept. 2001). This search yielded a total population of $n=377$ news articles and editorials. The entire population of articles was coded for three categorical attributes reflecting three distinct categories of attributes derived from the established framing literature.

The current study measured both affective and cognitive frames derived to reflect the diversity of content coding dominant in the framing literature (McCombs \& Ghanem, 2001). Affective frames largely measure the overall positive or negative tone of a story. Cognitive frames primarily identify three categories of substantive traits: ideology, qualifications, and personality (McCombs \& Ghanem, 2001). Categorical data coding enabled higher reliability among coders. To capture all the above information, we measured the following three categorical attributes:
$A_{1}=\{$ Tone $\}$

$A_{2}=\{$ Friend or Foe $\}$

$A_{3}=\{$ Reliability $\}$

"Tone" represents the traditional affective frame of the valence of an article's overall stand toward the individual, and it is measured as positive (favourable), negative (unfavourable), or neutral.

"Friend or Foe" is a cognitive frame that evaluates the degree to which Sharon and Arafat (and their relevant nation-states) are portrayed as embracing values and ideologies congruent to those of the United States.

The "Reliability" attribute represents the frame that incorporates expertise and personality traits related to the represented competency, credibility, stability, and leadership of the two individuals. This attribute signifies the extent to which each leader was represented as possessing authority over his people, engaging effectively with other nations, and behaving consistently and reliably toward foreign colleagues. Mentions of trustworthiness and strength of character prompted positive coding for "reliability". Reporting on a leader's deceptiveness, vacillation, and weakness yielded a "no" in this category. When an article described either Arafat or Sharon as a person whom foreign leaders did not take seriously or with whom they were unwilling to deal, as losing authority, as unreliable, incompetent, or not in control, the coders selected the "no" category. If none of the above elements appeared, the article was to be coded in the "yes" category. While coding for reliability, the authors measured both qualifications and personality attributes. This attribute taps into both qualifications and personality traits of the two leaders.
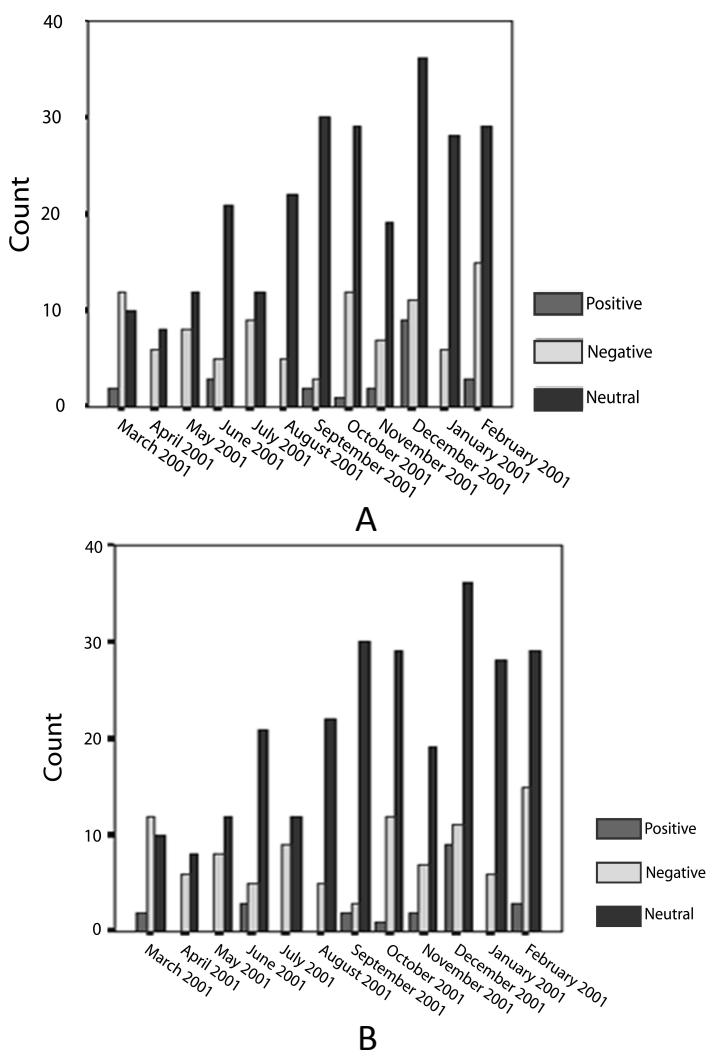

Fig. (1). Data for the "Tone" attribute for: (a) Arafat, and (b) Sharon. 
To this end, the domains of values (categories) for each attribute are given as follows:

$\operatorname{DOM}\left(\mathrm{A}_{1}\right)=\{$ Positive, Negative, Neutral $\}$

$\operatorname{DOM}\left(\mathrm{A}_{2}\right)=\{$ Friend, Foe, Neutral or N/A $\}$

$\operatorname{DOM}\left(A_{3}\right)=\{$ Yes, No $\}$

where N/A stands for "Not Applicable". Since the above domains of values do not appear in hierarchical structure, the obtained data are nominal categorical data.

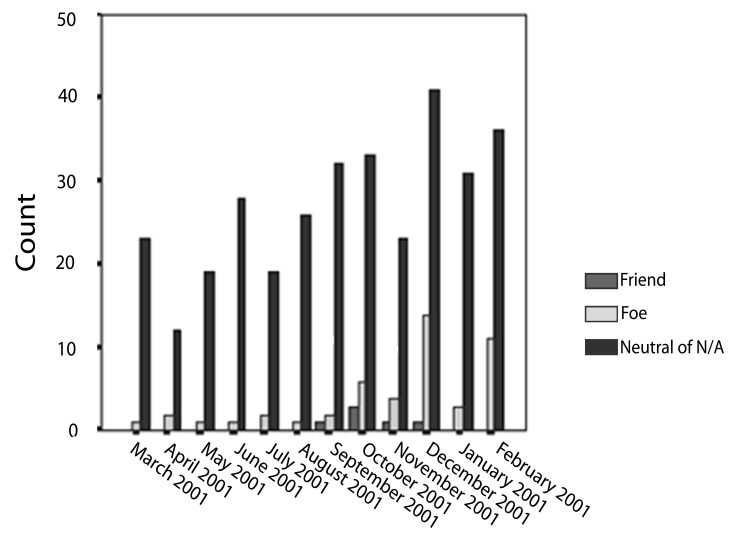

A

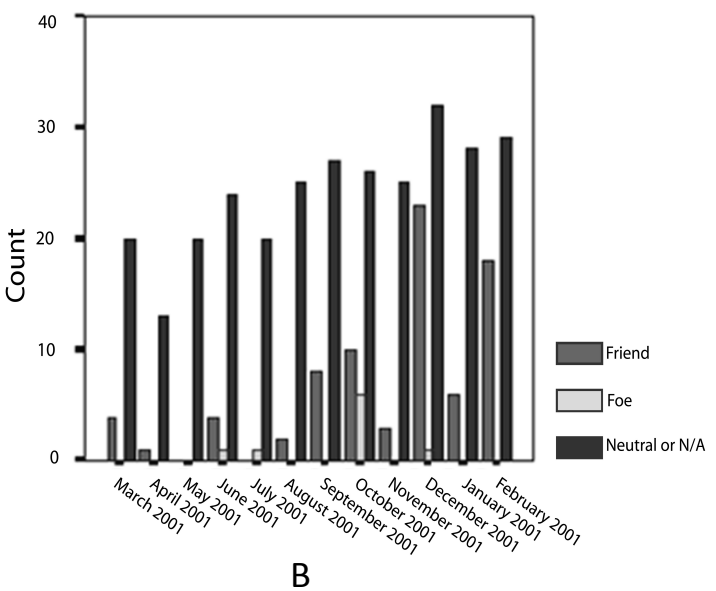

Fig. (2). Data for the "Friend or Foe" attribute for: (a) Arafat, and (b) Sharon.

To quantitatively describe the structure of the categorical data set we proceed with the following mathematical formulation:

The data set of the $n=377$ elements is denoted as $\mathbf{X}=\left\{\mathbf{x}_{1}, \mathbf{x}_{2}, \ldots, \mathbf{x}_{n}\right\}$, where $\mathbf{x}_{k}(k=1,2, \ldots, n) \quad$ is the $k$-th categorical element, that corresponds to the $k$-th article. Since each article was coded with respect to the three attributes, it is obvious that it was assigned three values each of which was taken from the respective domains of values given in equations (4)-(6). Therefore, the $k$-th categorical element is a single point in the three-dimensional space defined by the attributes $A_{1}, A_{2}$ and $A_{3}$, and it can be represented as,

$\mathbf{x}_{k}=\left[x_{k}^{1}, x_{k}^{2}, x_{k}^{3}\right]$

where

$x_{k}^{1} \in \operatorname{DOM}\left(A_{1}\right), x_{k}^{2} \in D O M\left(A_{2}\right)$ and $x_{k}^{3} \in \operatorname{DOM}\left(A_{3}\right)$.

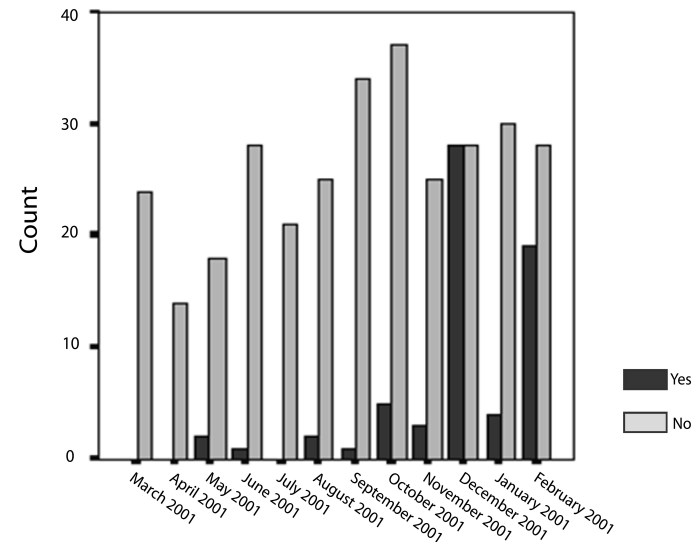

(a)

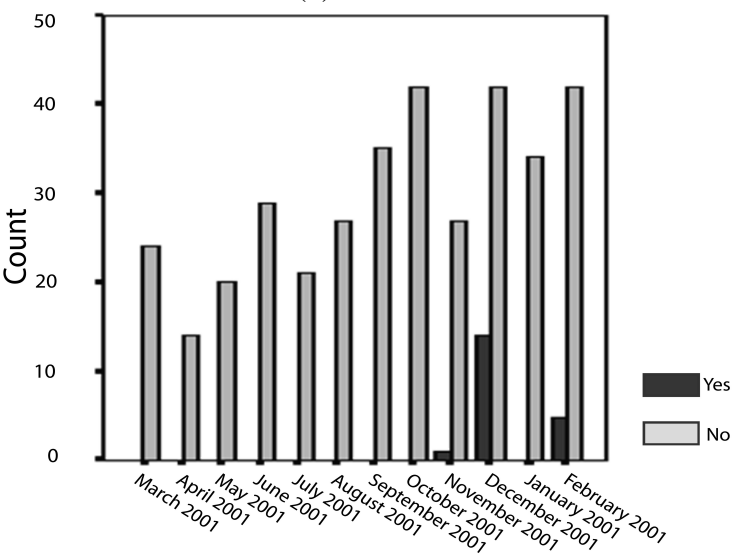

(b)

Fig. (3). Data for the "Reliability" attribute for: (a) Arafat, and (b) Sharon.

An example of a categorical element is:

$\mathbf{x}_{k}=\left[x_{k}^{1}, x_{k}^{2}, x_{k}^{3}\right]=[$ Positive, Foe, No $]$

For all cross-tabulations, chi-square tests were calculated, and they were statistically significant at 0.05 level, which social science researchers have established as a standard parameter (Holsti, 1969). Figs. (1-3) depict the available data sets for Arafat and Sharon in terms of the frequencies of categories' appearances in each month. Inter-coder reliability was measured for the coding by two independent, coders with expertise in quantitative content analysis. Holsti tests were calculated for all categorical variables. The degrees of agreement between the two coders were: "Tone toward Arafat" $=.79$, "Tone toward Sharon" = .77, "Arafat - Friend or Foe of the US" =.95, "Sharon - Friend or Foe of the US" $=.94$, "Reliability of Arafat" $=.79$, and "Reliability of Sharon"= .85 .

\section{STATISTICAL DATA ANALYSIS USING FUZZY CLUSTERING}

Rich analysis of the categorical data was challenged by the limited statistical options available to social scientists, particularly when different variables are examined in relation to one another. To reduce these difficulties and increase analytic insights, the authors employed categorical data clustering (CDC) to treat variables in a composite fashion rather than independently. Using $\mathrm{CDC}$ to construct composite frames, we clustered each leader's loyalty and 
affiliations with the United States, and their authority. The CDC method used here was developed by Tsekouras et al. (2005) and is based on fuzzy logic.

The main difference between classical logic and fuzzy logic is the following remark (Klir \& Bo, 1995): In classical (i.e., crisp) logic an element $x$ either belongs to the set $A$ or does not. That is, the membership degree of $x$ to $A$ is given as,

$$
u_{A}(x)= \begin{cases}1, & \text { if } x \in A \\ 0, & \text { otherwise }\end{cases}
$$

Hence, the answer to the question of whether an element belongs to a set or not is true or false. To the contrary, in fuzzy logic the membership degree of $x$ to the set $A$ takes the full range of values between 0 and 1 ,

$u_{A}(x) \in[0,1]$

In the latter case, the set $A$ is called a fuzzy set (Klir \& Bo, 1995). Based on the relation (9), a specific element may belong to more than one fuzzy set, with different membership degrees, where the sum of these degrees equals unity.

In cluster analysis, we are interested in partitioning an original data set of measurements coming from a real system (which may be a social system) into a number of clusters, where elements of the original set that belong to the same cluster are as similar as possible, whereas elements belonging to different clusters are as dissimilar as possible (Everitt, 1993). Therefore, the clusters are subsets of the original data set. The representative of each cluster is its center element, which does not belong to the original data set. The center element can be interpreted as the center of gravity (or the mean value) of the elements that belong to that cluster. The main intent of cluster analysis is to implement a specific algorithm in order to obtain the values for the cluster centers. These values indicate the final partition of the original data set. In the case of fuzzy cluster analysis, the clusters are called fuzzy clusters or fuzzy subsets.

The incorporation of fuzziness into the statistical analysis provides a flexible representation of the data set because the data elements belong to more than one fuzzy cluster with different participation degrees (Pedrycz \& Kwak, 2006). This directly suggests that fuzzy clustering is able to model the vagueness (or uncertainty) in a dataset (Novak, 2006; Liu, Wang, \& Chai, 2005). Typically, social data exhibit vagueness.

Clustering coded categories enables researchers to assess characteristics in a composite manner. The coded frames are directly evaluated as holistic framing combinations. It is this additional analysis through fuzzy CDC that adds to previous research and enhances the overall reliability of frame analysis and frame-shifting measurement. It is important to restate the limited utility of traditional social science statistical analyses for interpreting categorical data that may involve multiple interactions among categories. Fuzzy rather than crisp clustering of categorical data offers additional benefits. Specifically, one of the main characteristics that distinguishes fuzzy from crisp clustering is its inherent ability to model vagueness. Since coding involves to some degree content interpretation, the assessed frames of Arafat and Sharon include the element of vagueness inherent in interpretation. Therefore, we used fuzzy clustering to produce non-sharply defined clusters that would more accurately model the coding decisions.

To accomplish this, we put all three of the attributes of equations (1)-(3) into a three-dimensional space. Thus, we come up with a multidimensional cluster analysis of the data set. This analysis will demonstrate how each of the above attributes affects the others and how this affects the results in possible tradeoffs among the different variables. This application cannot be accomplished through classical statistics such as log-linear or chi-square analyses, which usually are applied in one- dimensional data sets. Moreover, cluster analysis easily detects multidimensional structures in the data set, enabling a qualitative interpretation of these structures (Everitt, 1993).

To this end, the steps of the proposed statistical analysis are described in detail below. The method is applied to only one leader at a time.

Step 1). We apply fuzzy cluster analysis to the data set, described in the previous section, in order to produce a number of fuzzy clusters in the three-dimensional space defined by the attributes $A_{1}, A_{2}$ and $A_{3}$. Let us denote the number of the produced fuzzy clusters with the letter $c$, and the respective center elements as $\boldsymbol{V}_{i}$ with $i=1,2, \ldots ., c$.

Step 2). Each one of the cluster centers is a point (i.e. , an element) in the three-dimensional space. Therefore, it has three coordinates and it can be represented as,

$\mathbf{v}_{i}=\left[v_{i}^{1}, v_{i}^{2}, v_{i}^{3}\right](i=1,2, \ldots . ., c)$

where again,

$$
v_{i}^{1} \in \operatorname{DOM}\left(A_{1}\right), v_{i}^{2} \in \operatorname{DOM}\left(A_{2}\right) \text { and } v_{i}^{3} \in \operatorname{DOM}\left(A_{3}\right) .
$$

The coders used the coordinate values $v_{i}^{1}, v_{i}^{2}$ and $v_{i}^{3}$ to assign a label to the $i$-th cluster center, which qualitatively interprets the semantics of the respective fuzzy cluster and therefore, this label is referred (i.e. , reflected) to the whole fuzzy cluster. For example if the center element of the $i$-th fuzzy cluster is,

$$
\mathbf{v}_{i}=\left[v_{i}^{1}, v_{i}^{2}, v_{i}^{3}\right]=[\text { Negative }, \text { Foe }, \text { No }]
$$

then the respective label for this fuzzy cluster could be "Negative" or "Very Negative". Thus, we conclude that this fuzzy cluster represents the negative voices with respect to the picture of the leader.

Step 3). We determine the weight of significance of each cluster in a specific month. The weight of significance of the $i$-th fuzzy cluster in the $l$-th month $(l=1,2, \ldots, 12)$ is estimated according to the following formula,

$\pi_{i}^{\prime}=\frac{N_{i}^{\prime}}{\aleph^{\prime}}$

where,

$N_{i}^{\prime}$ : The number of categorical elements that simultaneously belong to the $i$-th fuzzy cluster and the $l$-th month.

$\aleph^{\prime}$ : The total number of categorical elements that belong to the $l$-th month. 
In terms of fuzzy logic the equation (11) is rewritten using the concept of the membership degree as follows,

$\partial_{i}^{l}=\sum_{\boldsymbol{x}_{k} \in X_{l}} u_{i k} / \sum_{i=1}^{c} \sum_{\boldsymbol{x}_{k} \in X_{l}} u_{i k}(1 \leq i \leq c, 1 \leq l \leq 12)$

where $\boldsymbol{X}_{l}$ is the number of categorical elements that belong in the $l$-th month with $\boldsymbol{X}=\bigcup_{l=1}^{12} \boldsymbol{X}_{l}$.

Thus, the eq. (11) and (12) are equivalent. From eq. (11) or (12) we can easily verify that $\pi_{i} \in[0,1]$ and for the $l$-th month,

$$
\pi_{1}^{\prime}+\pi_{2}^{\prime}+\ldots+\pi_{c}^{\prime}=1
$$

To perform the fuzzy clustering in step 1 of the above method we used the algorithm developed by Tsekouras et al. (2005), which is based on the fuzzy c-modes algorithm (Huang and $\mathrm{Ng}, 1999$ ). The interested reader can find a detailed analysis in these two references. The determination of the weights in step 3 provides certain advantages such as:

- Detection of any possible dynamic change (i.e., shifting) of the fuzzy clusters during this period.

- Detection of any possible tradeoff between the fuzzy clusters.

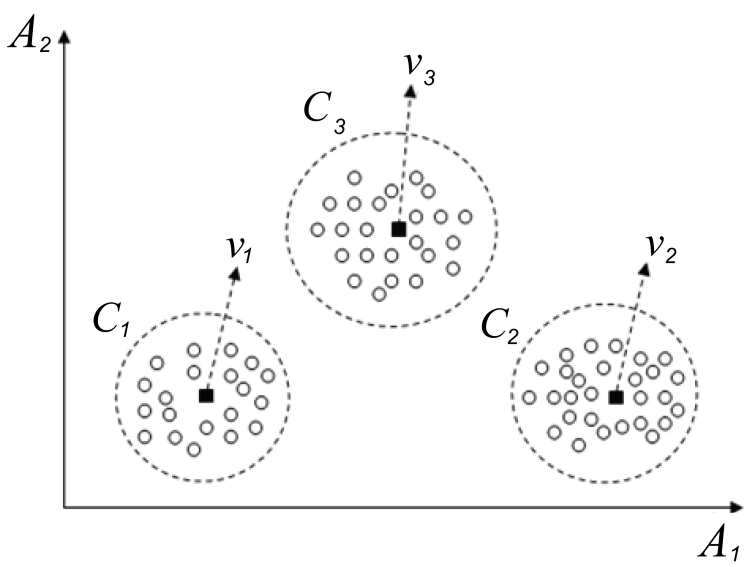

Fig. (4). Distribution of the elements and fuzzy cluster characteristics for the hypothetical example.

To demonstrate the functionality of the above method we proceed with a hypothetical example. To simplify the whole process, we assume that we have only two attributes $A_{1}$ and $A_{2}$. Therefore, we use two-dimensional space. Moreover, since categorical data are difficult to visualize, we assume that the available data are continuous elements. Fig. (4) shows a possible scenario. In this figure, the elements of the original data set are denoted by the circles. In total, there are $n=72$ categorical elements.

The implementation of the fuzzy clustering algorithm will finally produce $c=3$ fuzzy clusters, the centers of which are denoted as $\boldsymbol{V}_{1}, \boldsymbol{V}_{2}$ and $\mathbf{v}_{3}$. In Fig. (4), the cluster centers are depicted by the bold rectangles. Based on the coordinates of the centers, we assign the labels $C_{1}, C_{2}$ and $C_{3}$ for the first, the second and the third fuzzy cluster, respectively.
Table 1. Distribution of the Elements in a 4-Month Period for the Hypothetical Example

\begin{tabular}{|c|c|c|c|c|}
\hline Month & $C_{1}$ & $C_{2}$ & $C_{3}$ & Total \\
\hline \hline 1 & 9 & 6 & 3 & 18 \\
\hline 2 & 6 & 9 & 6 & 21 \\
\hline 3 & 3 & 6 & 7 & 16 \\
\hline 4 & 2 & 7 & 8 & 17 \\
\hline
\end{tabular}

Table 2. The Final Weights of Significance in a 4-Month Period for the Hypothetical Example

\begin{tabular}{|c|c|c|c|c|}
\hline Month $(\boldsymbol{l})$ & $\boldsymbol{\pi}_{1}^{l}$ & $\boldsymbol{\pi}_{2}^{l}$ & $\boldsymbol{\pi}_{3}^{l}$ & Total \\
\hline \hline $\boldsymbol{l = 1}$ & 0.500 & 0.333 & 0.167 & 1 \\
\hline $\boldsymbol{l = 2}$ & 0.286 & 0.428 & 0.286 & 1 \\
\hline $\boldsymbol{l}=\mathbf{3}$ & 0.187 & 0.375 & 0.438 & 1 \\
\hline $\boldsymbol{l}=\mathbf{4}$ & 0.118 & 0.411 & 0.471 & 1 \\
\hline
\end{tabular}

Now, let us assume that the distribution of the original categorical elements within a 4-month period is given in the last column of Table 1. Moreover, this table indicates how many elements of each fuzzy cluster are reported in each month. Therefore, this table also indicates the distribution of the elements of each fuzzy cluster during the 4-month period.

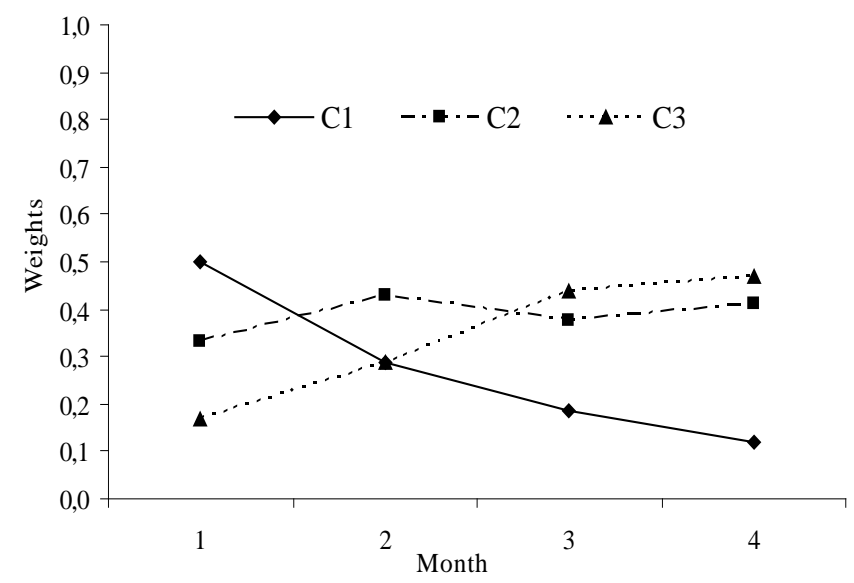

Fig. (5). Dynamic change of the weights for each fuzzy cluster for the case of the hypothetical example.

Then, using eq. (12), we can easily estimate the weight of significance of each cluster with respect to specific months. For example, the weight of second fuzzy cluster in the third month is equal to $6 / 16=0.375$. The resulting weights of significance are given in Table 2 . In this table, the sum for the elements of each raw equals unity, as it is stated in eq. (13). If we want to interpret the above weights, we can say that the weight $\pi_{i}^{l}$ reflects the relative significance of the $i$ th fuzzy cluster in the $l$-th month. Note that the weight of each cluster is calculated relatively to the rest of the clusters, something which is very useful when we want to detect and study possible interactions among them. The visualization of Table $\mathbf{2}$ is given in Fig. (5), which shows how the weights of significance for each fuzzy cluster change (shift) with time. 
At this point, we emphasize that the above method is not specially designed for the current case study, but rather it can be implemented in any multidimensional case where dynamic changes must be studied and interpreted.

\section{RESULTS}

The application of the fuzzy cluster analysis (i.e., the step 1 of the method) gave $c=5$ and $c=4$ fuzzy clusters for Arafat and Sharon, respectively. Tables 3 and $\mathbf{4}$ depict the final cluster centers for these two cases.

Table 3. Final Fuzzy Cluster Centers for the Arafat's Case and the Respective Labels

\begin{tabular}{|c|c|c|c|c|}
\hline $\mathbf{i}$ & $\mathbf{A}_{\mathbf{1}}$ & $\mathbf{A}_{\mathbf{2}}$ & $\mathbf{A}_{\mathbf{3}}$ & Portrayal Label \\
\hline \hline 1 & Positive & Neutral or N/A & No & Positive \\
\hline 2 & Neutral & Neutral or N/A & No & Neutral \\
\hline 3 & Negative & Neutral or N/A & No & Negative \\
\hline 4 & Negative & Neutral or N/A & Yes & Very Negative \\
\hline 5 & Negative & Foe & No & Strong Negative \\
\hline
\end{tabular}

Table 4. Final Fuzzy Cluster Centers for the Sharon's Case and the Respective Labels

\begin{tabular}{|c|c|c|c|c|}
\hline $\mathbf{i}$ & $\mathbf{A}_{\mathbf{1}}$ & $\mathbf{A}_{\mathbf{2}}$ & $\mathbf{A}_{\mathbf{3}}$ & Portrayal Label \\
\hline \hline 1 & Positive & Friend & Yes & Strong Positive \\
\hline 2 & Neutral & Friend & No & Very Positive \\
\hline 3 & Neutral & Neutral or N/A & No & Neutral \\
\hline 4 & Negative & Neutral or N/A & No & Negative \\
\hline
\end{tabular}

Adopting a different point of view, the above fuzzy clusters are patterns, each of which corresponds to a composite (i.e., multi-dimensional) frame. These composite frames define the values that are assigned to the overall media picture of the respective person, and they thus are called portrayal patterns. Thereby, the words "portrayal pattern" and "fuzzy cluster" have the same meaning. According to step 2 of the method, we can obtain an interpretation of the portrayal patterns of the two leaders by performing a uniform labeling assessment. To assign the portrayal labels, we took into account the degree of convergence among variables. For example, if all variables had been coded as "positive", the respective portrayal pattern would be labeled as more "positive" than a coding assessment in which only two of the three variables were coded as positive. For distribution of these labels see the last columns of Tables 3 and $\mathbf{4}$. The labels assigned in these tables are very helpful in extracting qualitative results with respect to the overall media pictures of the two leaders.

The implementation results concern the dynamic changes of the portrayal patterns in the 12-month period. To determine the dynamic changes of the patterns, we apply the step 3 of the method. Each portrayal pattern is comprised of a number of categorical elements. Moreover, these categorical elements may belong to different months in the 12-month timeline. Thus, in the context of the 12-month period, each portrayal pattern may share common elements across more than one month. Therefore, each portrayal pattern is assigned weights, using the eq. (12)

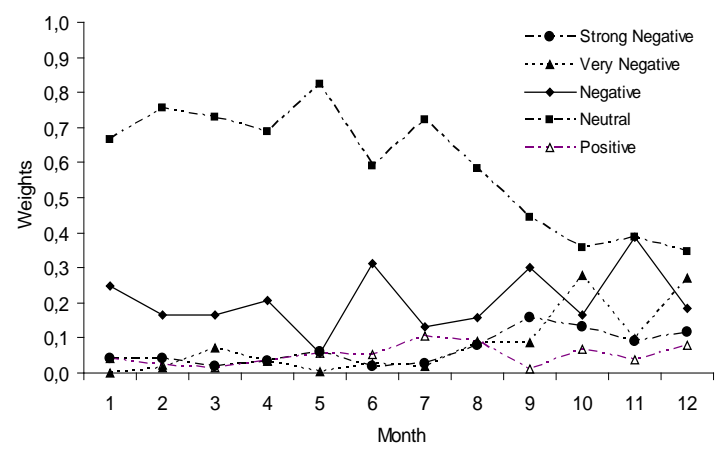

(a)

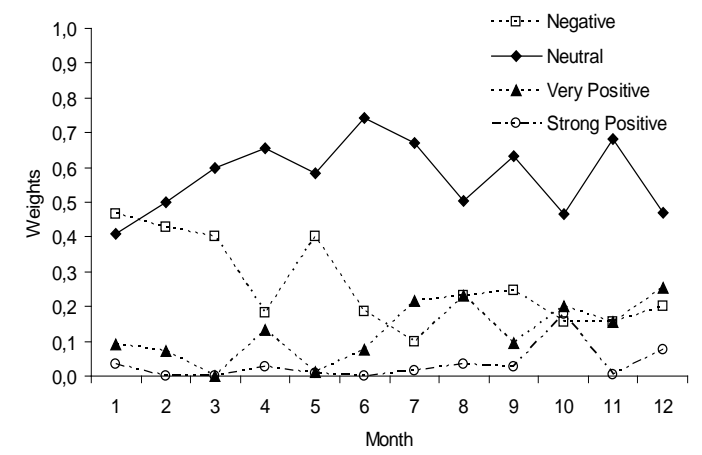

(b)

Fig. (6). Changing portrayal patterns of Arafat (a) and Sharon (b) as a function of time.

Based on the above analysis, any change of the portrayal patterns is reflected in shifts in the respective weights. Therefore, the weights provide a qualitative measure of the portrayal patterns' shift in time and are called shifting portrayal patterns. Fig. (6) shows the shifting portrayal patterns of Arafat and Sharon. These two figures support the authors' hypothesis that media frames would change significantly after the cataclysmic event of Sept. 11, 2001. As (Fig 6a) demonstrates, in Arafat's case negative frames increased after Sept. 11, while neutral coverage dropped significantly. These two countervailing changes suggest a tradeoff between neutral and negative voices, while positive coverage remains steady throughout the whole year.

According to (Fig. 6b), in Sharon's case negative coverage decreased significantly following Sept. 11, while positive coverage increased. Extreme cases, such as the "Strong Positive" coverage, remained fairly constant throughout the whole 12-month period as did the amount of neutral coverage. On the other hand, greater fluctuations during the second six-month period indicate greater coverage variability of Sharon after Sept. 11.

\section{CONCLUSIONS}

The results of this study and the application of fuzzy logic clustering analysis demonstrate significant shifts in media coverage of Israel and Palestine during the post-Sept. 11 period. The data show that The New York Times did not 
consistently apply a dominant frame to either nation-state leader. Rather, during the period of strong elite consensus in support of the U.S. government policy against terrorism following Sept. 11, the newspaper's treatment of the ongoing conflict in the Middle East became more varied and multifaceted. The newspaper also covered the region more intensely in the six months after September 2001. As Entman's theory would predict, the varied and conflicting frames may embody the more complicated worldview adopted by increasingly independent media during the postCold War era (Entman, 2004).

Another possible explanation for the erratic frame shifts and more complex framing during the post-Sept. 11 period is that the frames of Arafat and Sharon are the product of elite friction and continued instability in U.S. policy toward the Middle East, as Wolfsfeld (2004) might suggest. It is also possible that the greater range of frames presented following Sept. 11 is the rather predictable result of the increased frequency of coverage. Shinar (2003) would also suggest that shifting coverage by The New York Times might reflect the "crisis of expectations" that arises when policy objectives for peace confront fundamentally intractable cultural conflicts.

While this study cannot determine the causes of the observed changes, it does demonstrate that the newspaper's portraits of Arafat and Sharon are not the relatively static and simplistic representations envisioned by framing theory. In place of impoverished, stereotypical, directive frames, the newspaper provided increasingly rich "resources of the imagination" from which individuals could construct their own reality (Anderson, 1991, Appadurai, 1997 \& Shah \& Thornton, 2004). As such, the newspaper provided opportunities for readers to see the region, its problems, and the possible solutions in new ways.

This study empirically supports theoretical perspectives that major events produce shifts in the relatively stable content frames that characterize media coverage (Savitch, 2003; Hess, 1996; Reese \& Danielian, 1991). Before and after Sept. 11, the news coverage of each leader individually changed. After the "terrorist" event, coverage of Yasser Arafat became more negative, and coverage of Ariel Sharon became more positive. While the disparity in coverage of the two widened, the coverage of Sharon also vacillated more widely after Sept. 11 .

The findings of this study also support the two seemingly contradictory contentions that media offer neutral reportage on the world and that news coverage is ideologically biased and pro-Israeli. Here the preponderance of The New York Times reporting on Ariel Sharon and Yasser Arafat is neutral in tone. However, the non-neutral coverage of each leader consistently offers more positive coverage of Sharon and more strongly negative coverage of Arafat. This result suggests that the often-rancorous argument between journalists and scholars over media objectivity and bias may, in fact, be over whether the glass is half full or half empty.

The methodology used for the current analysis represents an attempt to incorporate fuzzy logic clustering into the analysis of social science data and the investigation of social, mediated problems and hypotheses. Such an implementation offers significant advantages to social scientists as it permits them to examine media frame constructions and influences in a composite manner and to trace frame movements across extended periods of time. Furthermore, this research project combines elements of different sciences and promotes interdisciplinary research endeavors. However, this study represents an initial attempt at social science data analysis through fuzzy logic techniques. More research is needed to refine this strategy and to investigate the full potential of similar approaches. Furthermore, while this study offers an initial identification of frame shifts and concludes that some shifts may be linked to cataclysmic events, triangulating the methodology utilized likely would enrich results. Research designs incorporating quantitative as well as qualitative approaches would provide enhanced validity in terms of the scope of the definitions and the complexity of the identified variables and would be extremely valuable.

Among important questions that remain to be answered are the following: How durable or permanent are the observed frame shifts? What characteristics coincide with any reversion of frames to their original state? If some frames change permanently while others do not, what factors co-occur with these differences? Companion studies of frame shifts and policy changes would have important implications for the analysis of foreign policy and political initiatives.

The common characteristic of all of the above questions is the ambiguity that affects both data selection and interpretation. In this study, fuzzy logic clustering seems to handle this ambiguity efficiently and to offer greater depth and detail in the analysis and greater insight into the results of the quantitative coding than has been afforded by the methods adopted in prior media framing research. However, the greatest strength of the fuzzy logic approach is its ability to combine variables/frames and to treat different frame categories as composite frames, allowing for the assessment of overall effects on media content.

\section{REFERENCES}

Anderson, B. (1991). Imagined communities: reflections on the origin and spread of nationalism. London: Verso.

Appadurai, A. (1997). Modernity at large: Cultural dimensions of modernity. Minneapolis: University of Minnesota Press.

Berkowitz, D. (1992). Non-routine new and newswork: Exploring a what-astory. Journal of Communication 42(1), 82-94.

Entman, R.M. (2004). Projections of powe: Framing news, public opinion, and U.S. foreign policy. Chicago: The University of Chicago Press.

Everitt, B. (1993). Cluster analysis. N.Y.: Halsted Press.

Gamson, W.A. (1992). Talking politics. New York: Cambridge University Press.

Hess, S. (1996). International news \& foreign correspondents. Washington, DC: The Brookings Institution.

Holsti, O.R. (1969). Content analysis for the social sciences and humanities. Reading: Addison Wesley.

Huang, Z., \& Ng, M.K. (1999). A fuzzy k-modes algorithm for clustering categorical data. IEEE Transactions on Fuzzy Systems 7(4), 446452 .

Kepplinger, H., \& Habermeier, J. (1995). The impact of key events on the presentation of reality. European Journal of Communication 10(3), 371-390.

Klir, G., \& Bo, Y. (1995). Fuzzy sets and fuzzy logic: theory and applications. N.J.: Prentice Hall.

Liu, X., Wang, W., \& Chai, T. (2005). The fuzzy clustering analysis based on AFS theory. IEEE Transactions on Systems, Man and Cybernetics -Part B 35(5), 1013-1027.

Mandelzis, L. (2003). The changing image of the enemy in the news discourse of Israeli newspapers, 1993-1994. Conflict \& Communication online 2(1). 
McCombs, M., \& Ghanem, S. (2001). The convergence of agenda setting and framing in framing public life: perspectives on media and our understanding of the social world, In: R.S.O. Gandy Jr. and A. Grant, (Eds). Framing public life. pp. 67-82. Mahwah, N.J.: Lawrence Erlbaum.

Novak, V. (2006). Which logic is the real fuzzy logic? Fuzzy Sets and Systems 157, 635-641.

Pedrycz, W., \& Kwak, K.C. (2006). Linguistic models as a framework of user-centric system modeling. IEEE Transactions on Systems, Man and Cybernetics-Part A 36(4), 727-745; Quarterly 61, 66.

Reese, S.D., \& Danielian, L.J. (1989). Intermedia influence and the drug issue: Convergence on cocaine. In: P.J. Shoemaker, (Ed.), Communication campaigns about drugs: Government, media and the public, (pp. 29-45). Hillsdale, NJ: Lawrence Erlbaum.

Rosenthal, U. (2003). September 11: Public administration and the study of crises and crisis management. Administration \& Society 35(2), 129.

Ross, S., \& Bantimaroudis, P. (2006). Frame shifts and catastrophic events: The attacks of September 11, 2001, and New York Times's portrayals of Arafat and Sharon. Mass Communication \& Society 9(1), 85-101.

Savitch, H.V. (2003). Does 9-11 portend a new paradigm for cities? Urban Affairs Review 39(1), 103-127.

Schlesinger, P., Elliot, P., \& Murdock, G. (1984). Televising terrorism: Political violence in popular culture. New York: Scribner.

Shah, H., \& Thornton, M.C. (2004). Newspaper coverage of interethnic conflict, competing visions of America. Thousand Oaks, CA.: Sage Publications.
Shin, B., Higa, K., Sheng O.R., \& Ide T. (1999). Analyzing the media usage behavior of telework groups: A contingency approach. IEEE Transactions on Systems, Man and Cybernetics-Part C 29(1), 127-139.

Shinar, D. (2003). Peace process in cultural conflict: The role of the media. Conflict \& Communication Online 2, 1.

Shoemaker, P.J. (1984). Media treatment of deviant political groups. Journalism Quarterly 61, 66.

Snow, D., \& Bedford, R. (1992). Master frames and cycles of protest. In: A. D. Morris \& C.M. Muller, (Eds.), Frontiers in social movement theory, (pp. 133-155). New Haven, CT: Yale University Press.

Steuter, E. (1990). Understanding the media/terrorism relationship: An analysis of ideology and the news in Time magazine. Political Communication and Persuasion 7, 257-278.

Tsekouras, G.E., Papageorgiou, D., Kotsiantis, S., Kalloniatis, C., \& Pintelas, P. (2005). A Fuzzy Logic-Based Approach for Detecting Shifting Patterns in Cross-Cultural Data. Lecture Notes in Artificial Intelligence 3533, 705-708.

Wolfsfeld, G. (1997). Media and political conflict: news from the Middle East. New York: Cambridge Univ. Press.

Wolfsfeld, G. (2004). Media and the path to peace. New York: Cambridge University Press.

Zadeh, L.A. (1971). Similarity relations and fuzzy orderings. Information Sciences 3, 177-200.

(C) Tsekouras et al.; Licensee Bentham Open.

This is an open access article licensed under the terms of the Creative Commons Attribution Non-Commercial License (http://creativecommons.org/licenses/by-nc/3.0/) which permits unrestricted, non-commercial use, distribution and reproduction in any medium, provided the work is properly cited. 